\title{
Energy, Oxidative Stress, and Inflammation in the Colon
}

\author{
Richard F. Harty
}

Published online: 25 August 2013

(c) Springer Science+Business Media New York 2013

The manuscript by Martinez et al. [1] describes the effects of topically applied $N$-acetylcysteine (NAC) on colonic histology and oxidative DNA damage in a rodent model of diversion colitis. The antioxidant NAC significantly improved histological inflammation scores in the diverted colon segments after 2 and 4 weeks of treatment. Furthermore, oxidative DNA damage in colonocytes was significantly reduced in segments without and with fecal stream exposure. These observations broaden our understanding of the role of oxidative stress in diversion colitis, and provide an opportunity to examine other interrelated factors involved in inflammation of the colon.

Energy metabolism in colonocytes is dependent on the luminal availability of short-chain fatty acids (SCFA), for example butyrate, propionate, and acetate [2]. Obligate anaerobic bacteria within the gut microbiome ferment and break down dietary complex carbohydrates and proteins to produce SCFA. Whereas glucose is a principal energy source for enterocytes in the small intestine, colonocytes are dependent on SCFAs to maintain energy homeostasis. Experimental and clinical studies support the concept that luminal deficiency of SCFAs, for example butyrate, exacerbate diversion colitis [3-5]. Clinical trials utilizing SCFA enemas to treat diversion colitis have been limited and inconclusive. Thus, because SCFA enemas are not used routinely to treat diversion colitis, surgical restoration of bowel continuity is definitive [5].

Butyrate regulation of energy metabolism in colonocytes has been studied in germfree (GF) mice [6]. Similar to humans, mouse colonocytes utilize bacterially-produced

R. F. Harty ( $₫)$

Altheus Therapeutics, Inc., 755 Research Parkway, Suite 435, Oklahoma City, OK 73104, USA

e-mail: Richard.Harty@altheustherapeutics.com butyrate as their primary energy source. In conventionally raised mice, butyrate is required for normal colonocyte ATP concentration. When gut flora is normal, microbiomegenerated butyrate is transported into colonocytes, entering the mitochondria, after which butyrate undergoes $\beta$-oxidation to acetyl-CoA, which then enters the tricarboxylic acid (TCA) cycle, reducing NAD+ to NADH. Upon entering the electron transport chain, $\mathrm{NADH}$ generates ATP and $\mathrm{CO}_{2}$. In the GF state, or likely in colonic diversion, colonocytes increase glucose uptake which increases the rate of glycolysis and lactate production at the expense of oxidative metabolism. Consequently, colonocyte ATP concentration is reduced because of inadequate butyrate availability. Nutrient-deficient and energy-deficient colonocytes will experience alterations in their redox state and decreased cellular oxidative phosphorylation with resultant increase in oxidative stress. In an effort to maintain energy homeostasis and cellular integrity, colonocytes in GF mice degrade cellular proteins and damaged organelles to generate amino acids for energy consumption in a process termed autophagy [6]. Addition of butyrate to GF colonocytes partially restores mitochondrial respiration and prevents autophagy. If these rescue efforts, for example autophagy, are not successful then cell death or apoptosis occurs. Recent research indicates that the process of autophagy is also important for clearing intracellular pathogens and may be an important component in immune surveillance and innate and adaptive gut immunity [7].

The ability of topically delivered NAC to ameliorate colitis in colon segments diverted from luminal bacteria and nutrients draws attention to the role of oxidative stress in the perpetuation of this pathologic condition. NAC is a watersoluble amino acid with L-cysteine and one acetyl group. The hydrogen atom in the sulfhydryl $(-\mathrm{SH})$ of sulfur-containing antioxidant molecules (thiols), including NAC and 
glutathione (GSH), acts as an electron source for neutralizing or reducing free radicals which contain one or more unpaired electrons on either oxygen (reactive oxygen species, ROS), or nitrogen (reactive nitrogen species, RNS) [8, 9]. Importantly, NAC also provides L-cysteine for synthesis of glutathione, an endogenous cellular antioxidant. Examples of oxygen-derived free radicals include superoxide $\left(\mathrm{O}_{2}^{--}\right)$and hydrogen peroxide $\left(\mathrm{H}_{2} \mathrm{O}_{2}\right)$; examples of nitrogen free radicals are peroxynitrite $\left(\mathrm{ONOO}^{-}\right)$and nitric oxide (NO). Free radicals are normally produced at low levels during cellular metabolism, particularly in the mitochondria, are shortlived, and are hydrogen-reduced by endogenous antioxidants, for example glutathione. Free radicals such as hydrogen peroxide and nitric oxide, produced enzymatically under stable conditions, mediate diverse physiological functions through cell signaling. Under conditions of oxidative stress, mitochondrial glutathione concentrations are diminished, increasing free radical production, inducing organelle dysfunction, autophagy, and resultant cytotoxicity [10].

Inflammation of the colon in experimental and clinical diversion colitis is characterized histologically by mucosal ulceration, crypt distortion, lamina propria infiltration by neutrophils, lymphocytes plasma cells and lymphoid hyperplasia $[1,4]$.

Appreciation of factors involved with initiation and perpetuation of inflammation in diversion colitis may relate, in part, to some of the issues previously mentioned, for example energy deprivation and oxidative stress. These factors may have implications to other forms of bowel inflammation.

Butyrate metabolism by mitochondria, under stable invitro conditions, generates low levels of ROS which, indirectly, inhibit the nuclear transcription pathway involving NF$\kappa \mathrm{B}$ [11]. Alternatively, the energy defect encountered in diversion colitis can lead to oxidative stress and high ROS concentrations, stimulating NF- $\kappa \mathrm{B}$ signaling, DNA binding, and subsequently, inflammatory cytokines production [10]. Mild increases in ROS stimulate production of another transcription factor, Nrf2, which translocates to the nucleus where it binds to the antioxidant response elements (ARE) present on stress responsive genes [10]. In turn, Nrf2 binding to ARE activates genes involved in cellular antioxidant and antiinflammatory defense mechanisms. Excessive ROS concentrations and oxidative stress interfere with Nrf2 translocation, enhancing NF- $\kappa \mathrm{B}$ signaling in an effort to protect the cells. This concentration and duration effect of biological substances, for example ROS and signaling proteins, is indicative of the duality of their potential actions and the importance of the regulatory systems needed to maintain homeostasis in cells and tissues.

Martinez and colleagues have also reported that 5-aminosalicylic acid (5-ASA) enemas in rats with diversion colitis effectively reduced microscopic mucosal injury and inflammation, and reduced oxidative DNA damage [12]. Moreover, 5-ASA functions as an antioxidant, a prostaglandin synthesis inhibitor, and a modulator of inflammatory cytokine production via the $\mathrm{NF}-\kappa \mathrm{B}$ pathway. Mesalamine, as topical or oral therapy, is a durable mainstay in frontline therapy for ulcerative colitis when formulated to deliver 5-ASA to sites of colonic inflammation. Because NAC and 5-ASA are pharmacologically similar, if one antioxidant is good might two be better in treating colitis? Combining the two antioxidants for topical delivery to the inflamed distal colon in a rodent model of colitis significantly and synergistically improves histological scores [13]. Moreover, simultaneous administration improved inflammatory biomarkers significantly more than with either agent used alone. Validation of the concept that the combination of NAC and 5-ASA is better than one for treating distal ulcerative colitis is now being addressed in clinical trials.

Targeted antioxidant therapy to specific detrimental reactive species at critical sites in a given disease state is an approach that has merit for future research. The targeted, topical delivery of NAC to the site of inflammation in diversion colitis may be one positive step in that direction. The complexities and binary functions of reactive species and transcription factors, for example, pose challenges for the investigator to find novel and effective approaches to diseases that develop as consequences of alterations in cellular energy, oxidative stress, and inflammation.

\section{References}

1. Martinez CAR, de Almeida MG, da Silva CMG, et al. Enemas with $n$-acetylcysteine can reduce the level of oxidative damage in cells of the colonic mucosa diverted from the faecal stream. Dig Dis Sci. (Epub ahead of print). doi:10.1007/s10620-013-2768-9.

2. Thibault R, Blachier F, Darcy-Vrillon B, et al. Butyrate utilization by the colonic mucosa in inflammatory bowel diseases: a transport deficiency. Inflamm Bowel Dis. 2010;16:684-695.

3. Pacheco RG, Esposito CC, Müller LC, et al. Use of butyrate or glutamine in enema solution reduces inflammation and fibrosis in experimental diversion colitis. World J Gastroenterol. 2012;18: 4278-4287.

4. Harig JM, Soergel KH, Komorowski RA, et al. Treatment of diversion colitis with short-chain-fatty acid irrigation. $N$ Engl J Med. 1989;320:23-28.

5. Nielsen OH, Vainer B, Rask-Madsen J. Non-IBD and noninfectious colitis. Nat Clin Pract Gastroenterol Hepatol. 2008;5: 28-39.

6. Donohoe DR, Garge N, Zhang X, et al. The microbiome and butyrate regulate energy metabolism and autophagy in the mammalian colon. Cell Metab. 2011;13:517-526.

7. Murphy SF, Kwon JH, Boone DL, et al. Novel players in inflammatory bowel disease pathogenesis. Curr Gastroenterol Rep. 2012;14:146-152. 
8. Zhang H, Forman HJ. Glutathione synthesis and its role in redox signaling. Semin Cell Dev Biol. 2012;23:722-728.

9. Circu ML, Aw TY. Intestinal redox biology and oxidative stress. Semin Cell Dev Biol. 2012;23:729-737.

10. Bolisetty S, Jaimes EA. Mitochondria and reactive oxygen species: physiology and pathophysiology. Int J Mol Sci. 2013; 14:6306-6344.

11. Kumar A, Wu H, Collier-Hyams LS, et al. The bacterial fermentation product butyrate influences epithelial signaling via reactive oxygen species-mediated changes in cullin-1 neddylation. J Immunol. 2009;182:538-546.
12. Caltabiano C, Máximo FR, Spadari AP, et al. 5-aminosalicylic acid (5-ASA) can reduce levels of oxidative DNA damage in cells of colonic mucosa with and without fecal stream. Dig Dis Sci. 2011;56:1037-1046.

13. Ancha HR, Kurella RR, McKimmey CC, et al. Effects of $N$ acetylcysteine plus mesalamine on prostaglandin synthesis and nitric oxide generation in TNBS-induced colitis in rats. Dig Dis Sci. 2009;54:758-766. 